\title{
OPEN Prevalence of celiac disease in low and high risk population in Asia-Pacific region: a systematic review and meta-analysis
}

\author{
Sara Ashtari ${ }^{1}$, Hadis Najafimehr ${ }^{1}$, Mohamad Amin Pourhoseingholi ${ }^{1}$, Kamran Rostami ${ }^{2}$, \\ Hamid Asadzadeh-Aghdaei ${ }^{3}$, Mohammad Rostami-Nejad ${ }^{1 \bowtie}$, Mostafa Rezaei Tavirani ${ }^{4}$, \\ Meysam Olfatifar ${ }^{3}$, Govind K. Makharia ${ }^{5}$ \& Mohammad Reza Zali ${ }^{1}$
}

This systematic review and meta-analysis study was conducted to estimate the pooled prevalence of $C D$ in low and high risk groups in this region. Following keywords were searched in the Medline, PubMed, Scopus, Web of Science and Cochrane database according to the MeSH terms; celiac disease, prevalence, high risk population and Asian-Pacific region. Prevalence studies published from January 1991 to March 2018 were selected. Prevalence of $C D$ with $95 \%$ confidence interval (Cl) was calculated using STATA software, version 14. The pooled sero-prevalence of $C D$ among low risk group in Asia-Pacific region was $1.2 \%(95 \% \mathrm{Cl} 0.8-1.7 \%)$ in 96,099 individuals based on positive anti-tissue transglutaminase (anti-t-TG Ab) and/or anti-endomysial antibodies (EMA). The pooled prevalence of biopsy proven $\mathrm{CD}$ in Asia-Pacific among high and low risk groups was $4.3 \%(95 \% \mathrm{Cl} 3.3-5.5 \%)$ and $0.61 \%(95 \% \mathrm{Cl} 0.4-0.8 \%)$ in 10,719 and 70,344 subjects, respectively. In addition, the pooled seroprevalence and prevalence of $C D$ in general population was significantly higher in children compared with adults and it was significantly greater in female vs. male $(P<0.05)$. Our results suggest high risk individuals of $C D$ are key group that should be specifically targeted for prevention and control measures, and screening may prove to have an optimal cost-benefit ratio.

Celiac disease (CD) is a chronic autoimmune disorder which characterized by inflammation and villous atrophy (VA) in the small intestine that affects people who are genetically predisposed ${ }^{1,2}$. Even though the prevalence of $\mathrm{CD}$ varies from region to region, the average prevalence of the disease has been reported between 0.5 and $1 \%$ worldwide ${ }^{3,4}$. Evidence suggests that CD is higher in patients with genetic and autoimmune diseases than in healthy individuals. Prevalence of CD is high in patients with insulin dependent diabetes mellitus type 1 (DM1), chronic diarrhea, autoimmune thyroid disease (ATD), autoimmune hepatitis, Down syndrome (DS), inflammatory bowel disease (IBD), irritable bowel syndrome (IBS), Turner syndrome (TS), and first-degree relatives (FDR) of patients with $\mathrm{CD}^{5,6}$.

Numerous studies have been conducted in various parts of the world ${ }^{7,8}$, on the prevalence of CD in the general population, including in the Asia-Pacific region ${ }^{4,9}$. A meta-analysis of prevalence of CD in general population amongst Asian has been conducted earlier by Singh et al. however there is no reported the prevalence of CD in high risk individuals in this region. Therefore we conducted this systematic review and meta-analysis to determine and compare the prevalence of $\mathrm{CD}$ in high risk (first-degree relatives of patients with $\mathrm{CD}$, patients with DS, DM1, ATD, IBD, dyspeptic and children and adults with symptoms frequently associated with CD such as; diarrhea and abdominal pain), and low risk (blood donors, schoolchildren and subjects without any diseases) population in Asia-Pacific region. Subgroup and meta-regression analysis were also used to address the heterogeneity between the studies in this meta-analysis.

\footnotetext{
${ }^{1}$ Gastroenterology and Liver Disease Research Center, Research Institute for Gastroenterology and Liver Diseases, Shahid Beheshti University of Medical Science, Tehran, Iran. ${ }^{2}$ Departments of Gastroenterology, Mid Central DHB, Palmerston Hospital, Palmerston North, New Zealand. ${ }^{3}$ Basic and Molecular Epidemiology of Gastrointestinal Disorders Research Center, Research Institute for Gastroenterology and Liver Diseases, Shahid Beheshti University of Medical Sciences, Tehran, Iran. ${ }^{4}$ Proteomics Research Center, Faculty of Paramedical Sciences, Shahid Beheshti University of Medical Sciences, Tehran, Iran. ${ }^{5}$ Department of Gastroenterology and Human Nutrition, All India Institute of Medical Sciences, New Delhi, India. ${ }^{\circledR}$ email: m.rostamii@gmail.com
} 


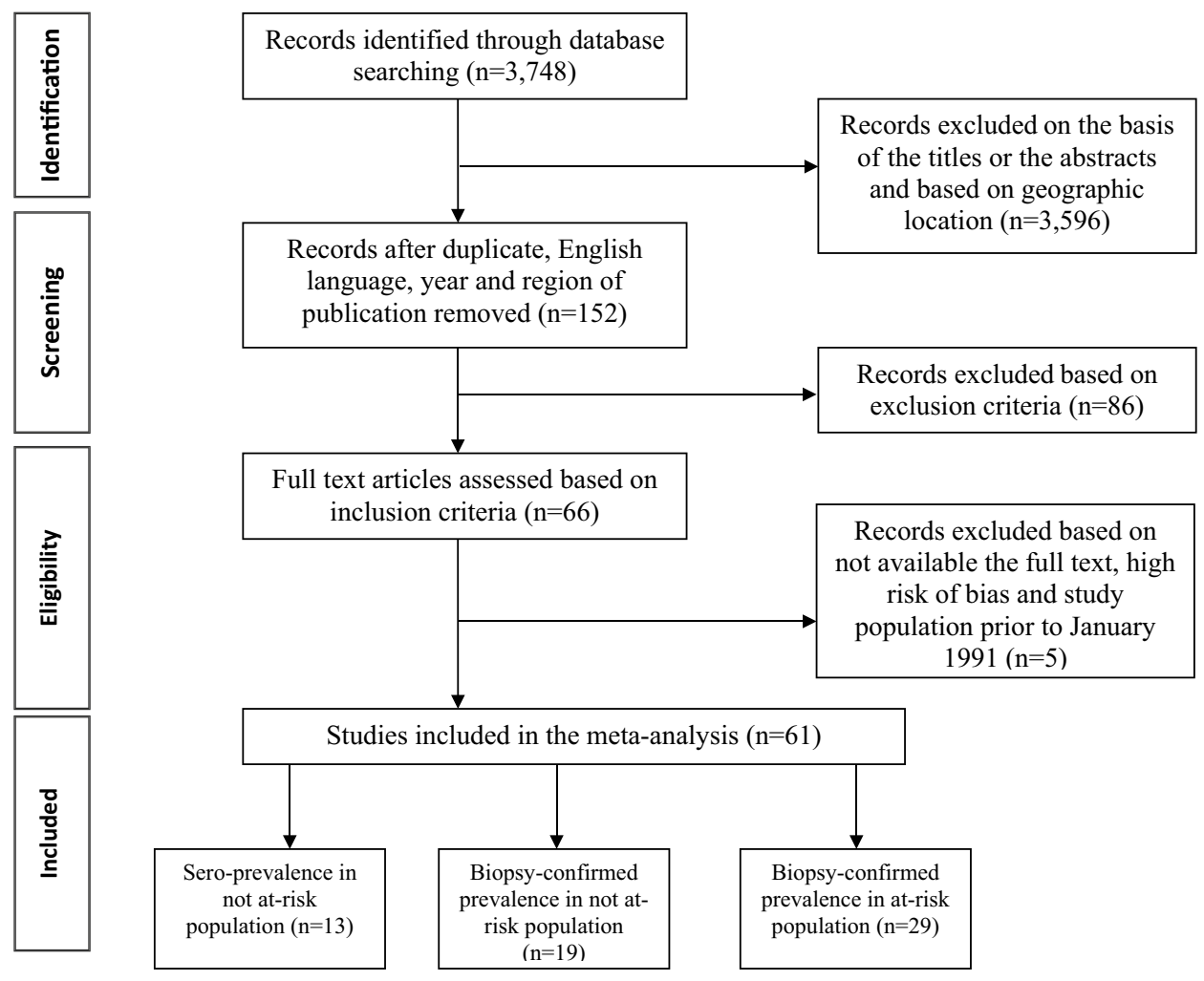

Figure 1. PRISMA flowchart of selecting the studies.

\section{Results}

Our search revealed a total of 3748 articles of CD prevalence in the database. Of them, 3596 articles were rejected on the basis of the titles or the abstracts and based on geographic location. Finally, full texts of 152 articles were assessed. Eighty-six additional studies were excluded based on the inclusion and exclusion criteria. Five more studies were excluded from the study because of; unavailable full texts, high-risk of bias and study conducted prior to January 1991. Ultimately, 61 studies were included in the present meta-analysis (Fig. 1). These 61 studies reporting the sero-prevalence and prevalence of $\mathrm{CD}$ among low and high risk population have originated from 13 Asian-Pacific countries; New Zealand, Australia, Turkey, India, Iran, Israel, Saudi-Arabia, Arab Emirates, Kuwait, Oman, Malaysia, China and Japan. Therefore, based on our findings, we divided these countries into four geographical categories; Oceania (New Zealand and Australia), Middle-East countries (Iran, Israel, Saudi-Arabia, Arab Emirates, Kuwait, Oman and Turkey), South-Asia (India and Malaysia) and East-Asia (China and Japan).

Pooled sero-prevalence of CD in low risk population. According to predefined criteria, 13 studies ${ }^{10-22}$ qualified for inclusion for estimation of the pooled sero-prevalence of CD (a positive EMA and/or antit-tTG Ab) in low risk population (Table 1). Of 96,099 included in 13 studies, 1,125 were reported to be seropositive for CD, suggesting a pooled sero-prevalence of CD to be $1.2 \%$ (95\% CI $\left.0.8-1.7 \%, \mathrm{I}^{2}=98 \%, P<0.01\right)$ (Fig. 2). The test of heterogeneity indicated a significant heterogeneity among the studies.

Age and gender-based difference in the sro-prevalence of CD among low risk group. Sero-prevalence of CD according to the gender was reported in 11 studies $^{10,12-19,21,22}$. Of 45,074 males and 37,597 females, 394 and 543 subjects had CD, respectively. Pooled sero-prevalence of CD in males and females were $0.8 \%$ (95\% CI $0.34-1.4 \%$, $\left.\mathrm{I}^{2}=97.9 \%, P=0.002\right)$ and $1.6 \%\left(95 \%\right.$ CI $\left.0.93-2.5 \%, \mathrm{I}^{2}=97 \%, P=0.002\right)$, respectively. The pooled sero-prevalence of CD was significantly higher in females than males $(P=0.04)$. In addition, the pooled sero-prevalence of CD was significantly higher in children as compared with adults $(2.04 \%$ vs. $0.95 \%, P<0.001)$.

Geographical difference in the sero-prevalence of $C D$ in low risk group. Of 13 studies that reported the seroprevalence of CD in Asia-Pacific region, 9 studies were from Middle East, 3 studies from South Asia and one study was from East Asia. The highest pooled sero-prevalence in Asia-Pacific region in the Middle-East $1.4 \%$ (95\% CI 0.9-2.1\%), and then in South-Asia 1.2\% (95\% CI 0.6-2.5\%) and the prevalence was least in East-Asia $0.06 \%$ (95\% CI $0.03-0.09 \%)$.

Pooled prevalence of biopsy-confirmed CD among low risk population. We found 19 studies $^{10-13,16,18,20,21,23-33}$ that reported the prevalence of biopsy-proven of CD among low risk population in AsiaPacific region (Table 2). Of 70,344 subjects, included in 19 studies, 472 were detected to have biopsy-proven 


\begin{tabular}{|c|c|c|c|c|c|c|c|c|c|c|c|}
\hline \multirow[b]{2}{*}{ First author } & \multirow[b]{2}{*}{ Country } & \multirow[b]{2}{*}{ Region } & \multirow[b]{2}{*}{ Year of study } & \multirow[b]{2}{*}{ Population } & \multirow[b]{2}{*}{ Sample size } & \multirow[b]{2}{*}{ Age (mean) } & \multirow{2}{*}{\begin{tabular}{|l|l}
$\begin{array}{l}\text { Serology } \\
\text { tests }\end{array}$
\end{tabular}} & \multirow[b]{2}{*}{ Risk of bias } & \multicolumn{3}{|c|}{ Sero-prevalence of CD (\%) } \\
\hline & & & & & & & & & Male & Female & Total \\
\hline Tatar $^{10}$ & Turkey & Middle East & $2001-2003$ & Adults & 2000 & 33 & $\mathrm{t}-\mathrm{TG} \mathrm{G}^{\mathrm{a}, \mathrm{b}}$ & Moderate & $22 / 1914(1.1)$ & $4 / 86(0.2)$ & $26 / 2000(1.3)$ \\
\hline $\begin{array}{l}\text { Saberi- } \\
\text { Firouzi }^{11}\end{array}$ & Iran & Middle East & 2004 & Adults & 1440 & 45.5 & $\begin{array}{l}\mathrm{t}-\mathrm{TG}^{\mathrm{a}} \\
\mathrm{EMA}^{\mathrm{a}}\end{array}$ & Low & - & - & $7 / 1,440(0.48)$ \\
\hline Ertekin $^{12}$ & Turkey & Middle East & 2005 & Children & 1263 & 11.9 & $t-T^{a}$ & Moderate & 6/687 (0.87) & $5 / 576(0.86)$ & \begin{tabular}{|l|l|}
$11 / 1,263$ \\
$(0.87)$
\end{tabular} \\
\hline Dalgic $^{13}$ & Turkey & Middle East & 2006-2008 & Children & 20,190 & 11.6 & $\begin{array}{l}\text { t-TG } \\
\text { EMA }^{\mathrm{a}, \mathrm{b}}\end{array}$ & Moderate & $\begin{array}{l}213 / 10,368 \\
(2.05)\end{array}$ & \begin{tabular}{|l|}
$276 / 9,822$ \\
$(2.81)$
\end{tabular} & $\begin{array}{l}489 / 20,190 \\
(2.42)\end{array}$ \\
\hline Aljebreen $^{14}$ & Saudi Arabia & Middle East & $2007-2008$ & Children & 1167 & 16.6 & $\mathrm{EMA}^{\mathrm{a}}$ & Low & 9/614 (1.46) & 17/553(3.07) & $26 / 1,167(2.2)$ \\
\hline Abu-Zeid ${ }^{15}$ & $\begin{array}{l}\text { Arab Emir- } \\
\text { ates }\end{array}$ & Middle East & $2007-2008$ & Adults & 1197 & 24.8 & $\begin{array}{l}\text { t-TG } \\
\text { EMA }^{a}\end{array}$ & Moderate & $1 / 624(0.16)$ & $13 / 573(2.27)$ & $\begin{array}{l}14 / 1,197 \\
(1.17)\end{array}$ \\
\hline Makharia $^{16}$ & India & South Asia & 2008-2009 & *both & 10,488 & 22.45 & $\mathrm{t}-\mathrm{TG}^{\mathrm{a}}$ & Moderate & $\begin{array}{l}68 / 5305 \\
(1.28)\end{array}$ & \begin{tabular}{|l|}
$83 / 5183$ \\
$(1.60)$
\end{tabular} & $\begin{array}{l}151 / 10,488 \\
(1.44)\end{array}$ \\
\hline Makharia $^{16}$ & India & South Asia & $2008-2009$ & Adults & 6845 & 34.4 & $\mathrm{t}-\mathrm{TG}^{\mathrm{a}}$ & Moderate & - & - & \begin{tabular}{|l|}
$75 / 6,845$ \\
$(1.10)$
\end{tabular} \\
\hline Makharia $^{16}$ & India & South Asia & 2008-2009 & Children & 3643 & 10.5 & $\mathrm{t}-\mathrm{TG}^{\mathrm{a}}$ & Moderate & - & - & \begin{tabular}{|l|}
$76 / 3,643$ \\
$(2.06)$
\end{tabular} \\
\hline Yuan $^{17}$ & China & East Asia & $2010-2013$ & Young Adults & 19,778 & 18.8 & $\begin{array}{l}\mathrm{t}-\mathrm{TG}^{\mathrm{a}} \\
\mathrm{DGP}^{\mathrm{b}}\end{array}$ & Moderate & \begin{tabular}{|l|}
$2 / 13,322$ \\
$(0.01)$
\end{tabular} & \begin{tabular}{|l|}
$9 / 6,456$ \\
$(0.14)$
\end{tabular} & \begin{tabular}{|l}
$11 / 19,778$ \\
$(0.06)$
\end{tabular} \\
\hline $\operatorname{Sezgin}^{18}$ & Turkey & Middle East & 2011-2013 & Adults & 1554 & 42.1 & $\begin{array}{l}\mathrm{t}-\mathrm{TG} G^{\mathrm{a}, \mathrm{b}} \\
\mathrm{DGP}^{\mathrm{a}, \mathrm{b}}\end{array}$ & Moderate & $2 / 772(0.12)$ & $10 / 782(0.64)$ & \begin{tabular}{|l|}
$12 / 1554$ \\
$(0.77)$
\end{tabular} \\
\hline $\begin{array}{l}\text { Ram- } \\
\text { akrishna }^{19}\end{array}$ & India & South Asia & 2011-2013 & Adults & 23,331 & 35 & $\mathrm{t}-\mathrm{TG}^{\mathrm{a}}$ & Low & \begin{tabular}{|l}
$58 / 10,776$ \\
$(0.5)$
\end{tabular} & $\begin{array}{l}100 / 12,555 \\
(0.8)\end{array}$ & $\begin{array}{l}158 / 23,331 \\
(0.68)\end{array}$ \\
\hline Dehghani $^{20}$ & Iran & Middle East & 2013 & \begin{tabular}{|l|} 
Children \\
\end{tabular} & 1500 & 9.5 & $\mathrm{t}-\mathrm{TG}^{\mathrm{a}}$ & Low & - & - & $30 / 1500(2)$ \\
\hline Hatlani $^{21}$ & Saudi Arabia & Middle East & 2012-2014 & \begin{tabular}{|l|} 
Children \\
\end{tabular} & 1141 & 11 & $\mathrm{t}-\mathrm{TG}^{\mathrm{a}}$ & Moderate & $12 / 454(2.6)$ & $20 / 687$ (3) & $32 / 1,141(2.8)$ \\
\hline Yap $^{22}$ & Malaysia & South Asia & 2012-2014 & Adults & 562 & 24 & $\begin{array}{l}\mathrm{AGA}^{\mathrm{a}, \mathrm{b}} \\
\mathrm{t}-\mathrm{TG}^{\mathrm{a}, \mathrm{b}} \\
\mathrm{EMA}^{\mathrm{a}, \mathrm{b}}\end{array}$ & Moderate & $1 / 238(0.4)$ & 6/324 (1.9) & $7 / 562(1.25)$ \\
\hline
\end{tabular}

Table 1. Sero-prevalence of CD in Asian-Pacific region among not at-risk population. EMA Anti-endomysial antibodies, $A G A$ Anti-gliadin antibodies, $t-T G$ tissue transglutaminase, DGP deamidated gliadin peptides. ${ }^{\mathrm{a}} \mathrm{IgA} .{ }^{\mathrm{b}} \mathrm{IgG}$. ${ }^{*}$ Adults and children together.

\section{Study}

Tatar 2003 Turkey Saberi-Firouzi 2004 Iran Ertekin 2005 Turkey Dalgic 2008 Turkey Aljebreen 2008 Saudi Arabia Abu-Zeid 2008 Arab Emirates Makharia 2009 India Makharia 2009 India Makharia 2009 India Yuan 2013 China Sezgin 2013 Turkey Ramakrishna 2013 India Dehghani 2013 Iran Hatlani 2014 Saudi Arabia Yap 2014 Malaysia

Random effects model Heterogeneity: $I^{2}=98 \%, \tau^{2}=0.0013, p<0.01$
Prevalence $95 \% \mathrm{CI}$

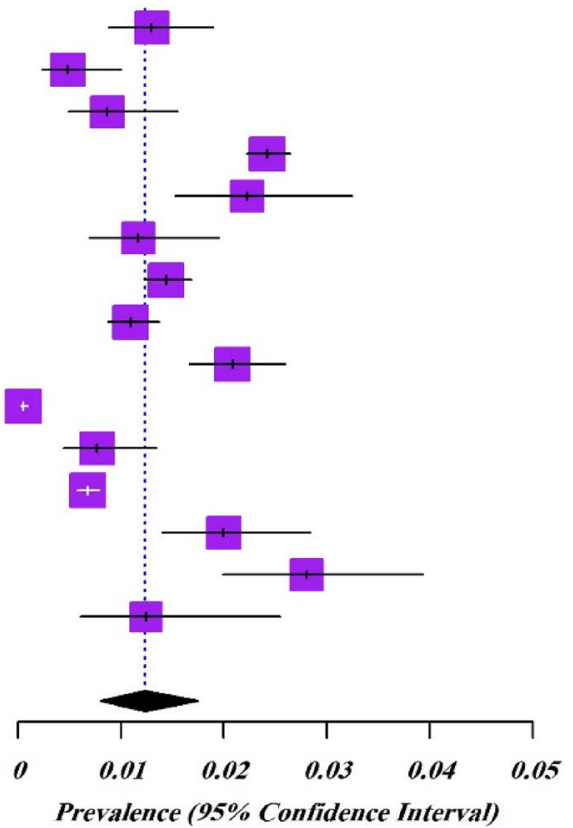

$\begin{array}{ll}0.01 & {[0.01 ; 0.02]} \\ 0.00 & {[0.00 ; 0.01]} \\ 0.01 & {[0.00 ; 0.02]} \\ 0.02 & {[0.02 ; 0.03]} \\ 0.02 & {[0.02 ; 0.03]} \\ 0.01 & {[0.01 ; 0.02]} \\ 0.01 & {[0.01 ; 0.02]} \\ 0.01 & {[0.01 ; 0.01]} \\ 0.02 & {[0.02 ; 0.03]} \\ 0.00 & {[0.00 ; 0.00]} \\ 0.01 & {[0.00 ; 0.01]} \\ 0.01 & {[0.01 ; 0.01]} \\ 0.02 & {[0.01 ; 0.03]} \\ 0.03 & {[0.02 ; 0.04]} \\ 0.01 & {[0.01 ; 0.03]}\end{array}$

0.01

$[0.01 ; 0.02]$

Figure 2. Forest plot for pooled sero-prevalence of CD in Asia-Pacific region among general population. 


\begin{tabular}{|c|c|c|c|c|c|c|c|c|c|c|c|}
\hline \multirow[b]{2}{*}{ First author } & \multirow[b]{2}{*}{ Country } & \multirow[b]{2}{*}{ Region } & \multirow{2}{*}{$\begin{array}{l}\text { Year of } \\
\text { study }\end{array}$} & \multirow[b]{2}{*}{ Population } & \multirow[b]{2}{*}{ Sample size } & \multirow[b]{2}{*}{ Age (mean) } & \multirow{2}{*}{$\begin{array}{l}\text { Serology } \\
\text { tests }\end{array}$} & \multirow[b]{2}{*}{ Risk of bias } & \multicolumn{3}{|c|}{ Prevalence of biopsy-confirmed (\%) } \\
\hline & & & & & & & & & Male & Female & Total \\
\hline $\mathrm{Cook}^{23}$ & New Zealand & Pacific & 2000 & Adults & 1064 & 50.2 & $\mathrm{EMA}^{\mathrm{a}, \mathrm{b}}$ & Moderate & $5 / 448(1.11)$ & $8 / 619(1.29)$ & $\begin{array}{l}13 / 1,064 \\
(1.22)\end{array}$ \\
\hline Hovell $^{24}$ & Australia & Pacific & 2001 & Adults & 3011 & - & EMA $^{\mathrm{a}}$ & Moderate & - & - & $7 / 3,011(0.23)$ \\
\hline Shamir ${ }^{25}$ & Israel & Middle East & $2000-2001$ & Adults & 1571 & 40.7 & $\begin{array}{l}\text { t-TGa } \\
\text { EMA }^{\mathrm{a}}\end{array}$ & Moderate & $8 / 1217(0.65)$ & $2 / 354(0.56)$ & $\begin{array}{l}10 / 1571 \\
(0.63)\end{array}$ \\
\hline $\begin{array}{l}\text { Shahbaz- } \\
\text { khani }^{26}\end{array}$ & Iran & Middle East & 2003 & Adults & 2000 & 35.5 & $\begin{array}{l}\text { AGA }^{\mathrm{a}} \\
\mathrm{EMA}^{\mathrm{a}}\end{array}$ & Moderate & \begin{tabular}{|l|}
$10 / 1580$ \\
$(0.63)$
\end{tabular} & $2 / 420(0.47)$ & $\begin{array}{l}12 / 2000 \\
(0.60)\end{array}$ \\
\hline Israeli $^{27}$ & Israel & Middle East & 2003 & Adults & 850 & 18 & $\mathrm{t}-\mathrm{TG}^{\mathrm{a}} \mathrm{EMA}^{\mathrm{a}, \mathrm{b}}$ & Moderate & - & - & $6 / 850(0.70)$ \\
\hline Tatar $^{10}$ & Turkey & Middle East & 2001-2003 & Adults & 2000 & 33 & $\mathrm{t}-\mathrm{TG}^{\mathrm{a}, \mathrm{b}}$ & Moderate & \begin{tabular}{|l}
$12 / 1914$ \\
$(0.62)$
\end{tabular} & $2 / 86(2.32)$ & $\begin{array}{l}14 / 2000 \\
(0.70)\end{array}$ \\
\hline $\begin{array}{l}\text { Saberi- } \\
\text { Firouzi }^{11}\end{array}$ & Iran & Middle East & 2004 & Adults & 1440 & 45.5 & $\mathrm{t}-\mathrm{TG}^{\mathrm{a}} \mathrm{EMA}^{\mathrm{a}}$ & Low & - & - & $2 / 1440(0.14)$ \\
\hline Akbari ${ }^{28}$ & Iran & Middle East & 2003-2004 & Adults & 2795 & 33.7 & $\begin{array}{l}\text { t-TGa } \\
\text { EMA }^{a}\end{array}$ & Low & $14 / 1398(1)$ & $13 / 1401(0.92)$ & $\begin{array}{l}27 / 2,799 \\
(0.96) \\
\end{array}$ \\
\hline Sood $^{29}$ & India & South Asia & $2003-2004$ & Children & 4347 & 10.7 & $\mathrm{t}-\mathrm{TG}^{\mathrm{a}}$ & Low & $4 / 2380(0.16)$ & $10 / 1,976(0.5)$ & $\begin{array}{l}14 / 4,347 \\
(0.32)\end{array}$ \\
\hline Ertekin $^{12}$ & Turkey & Middle East & 2005 & Children & 1263 & 11.9 & $\mathrm{t}-\mathrm{TG}^{\mathrm{a}}$ & Moderate & $4 / 687(0.58)$ & $3 / 576(0.52)$ & $7 / 1,263(0.55)$ \\
\hline Bahari $^{30}$ & Iran & Middle East & 2006-2007 & Adults & 1600 & 33.2 & $\mathrm{t}-\mathrm{TG}^{\mathrm{a}, \mathrm{b}}$ & Moderate & \begin{tabular}{|l|}
$14 / 1418$ \\
$(0.98)$
\end{tabular} & $0 / 182$ & $\begin{array}{l}14 / 1600 \\
(0.87)\end{array}$ \\
\hline Dalgic $^{13}$ & Turkey & Middle East & $2006-2008$ & Children & 20,190 & 11.6 & $\mathrm{t}-\mathrm{TG}^{\mathrm{a}} \mathrm{EMA}^{\mathrm{a}, \mathrm{b}}$ & Moderate & \begin{tabular}{|l}
$34 / 10,368$ \\
$(0.33)$
\end{tabular} & $\begin{array}{l}61 / 9,822 \\
(0.62)\end{array}$ & $\begin{array}{l}95 / 20,190 \\
(0.47)\end{array}$ \\
\hline Farahmand $^{31}$ & Iran & Middle East & $2006-2008$ & Children & 634 & \begin{tabular}{|l|}
12.8 \\
\end{tabular} & $\mathrm{t}-\mathrm{TG}^{\mathrm{a}}$ & Moderate & - & - & $3 / 634(0.47)$ \\
\hline Makharia $^{16}$ & India & South Asia & 2008-2009 & Both & 10,488 & 22.45 & $\mathrm{t}-\mathrm{TG}^{\mathrm{a}}$ & Moderate & \begin{tabular}{|l|l|}
$48 / 5,305$ \\
$(0.91)$
\end{tabular} & $\begin{array}{l}61 / 5,183 \\
(1.20)\end{array}$ & $\begin{array}{l}109 / 10,488 \\
(1.04)\end{array}$ \\
\hline Makharia $^{16}$ & India & South Asia & 2008-2009 & Adults & 6845 & 34.4 & $\mathrm{t}-\mathrm{TG}^{\mathrm{a}}$ & Moderate & - & - & $\begin{array}{l}58 / 6,845 \\
(0.85)\end{array}$ \\
\hline Makharia $^{16}$ & India & South Asia & 2008-2009 & Children & 3643 & 10.5 & $\mathrm{t}-\mathrm{TG}^{\mathrm{a}}$ & Moderate & - & - & $\begin{array}{l}51 / 3,643 \\
(1.41)\end{array}$ \\
\hline $\begin{array}{l}\text { Bhattacha- } \\
\text { rya }^{32}\end{array}$ & India & South Asia & 2009 & Children & 400 & 5.6 & $\mathrm{t}-\mathrm{TG}^{\mathrm{a}, \mathrm{b}}$ & Low & $1 / 228(0.4)$ & $3 / 172(1.7)$ & $4 / 400(1)$ \\
\hline $\operatorname{Sezgin}^{18}$ & Turkey & Middle East & 2011-2013 & Adults & 1554 & 42.1 & $\begin{array}{l}\mathrm{t}-\mathrm{TG}^{\mathrm{a}, \mathrm{b}} \\
\mathrm{DGP}^{\mathrm{a}, \mathrm{b}}\end{array}$ & Moderate & $0 / 772$ & $6 / 782(0.76)$ & $6 / 1,554(0.39)$ \\
\hline Dehghani $^{20}$ & Iran & Middle East & 2013 & Children & 1500 & 9.5 & $\mathrm{t}-\mathrm{TG}^{\mathrm{a}}$ & Low & $4 / 825(0.48)$ & $5 / 675(0.74)$ & $9 / 1500(0.6)$ \\
\hline Hatlani $^{21}$ & Saudi Arabia & Middle East & 2012-2014 & Children & 1141 & 11 & $\mathrm{t}-\mathrm{TG}^{\mathrm{a}}$ & Moderate & $4 / 454(0.9)$ & $6 / 687(0.9)$ & $10 / 1,141(0.9)$ \\
\hline Fukunaga $^{33}$ & Japan & East Asia & 2014-2016 & Adults & 2008 & 53 & $\begin{array}{l}\text { t-TGa } \\
\text { EMA }^{a}\end{array}$ & Moderate & $1 / 1351(0.07)$ & $0 / 657$ & $1 / 2008(0.05)$ \\
\hline
\end{tabular}

Table 2. Prevalence of biopsy-confirmed CD in Asia-Pacific region among not at-risk population.

CD suggestion a pooled prevalence of biopsy-proven CD among not at-risk population to be $0.61 \%(95 \% \mathrm{CI}$ $0.4-0.8 \%, \mathrm{I}^{2}=84 \%, P<0.01$ ) (Fig. 3A). The $\mathrm{I}^{2}$ test indicated significant heterogeneity among the studies.

Age and gender-based difference in the prevalence of biopsy-confirmed CD among not low risk group. In low risk population, eight studies reported prevalence of biopsy-confirmed CD in children, 12 studies in adults, and 1 study reported the prevalence in both adults and children combined. The pooled prevalence of CD in low risk children was $0.66 \%$ (95\% CI $\left.0.4-0.9 \%, \mathrm{I}^{2}=82.3 \%, P<0.001\right)$, in adults was $0.55 \%$ (95\% CI $0.3-0.8 \%$, $\left.\mathrm{I}^{2}=82.5 \%, P<0.001\right)$ and combined adults and children was $1.04 \%$ (95\% CI $\left.0.8-1.2 \%\right)$. The pooled prevalence of biopsy-confirmed CD among low risk population in children was significantly higher than adults $(0.6 \%$ vs. $0.5 \%$, $P=0.022)$. The $\mathrm{I}^{2}$ tests indicated similar heterogeneity among the studies reporting CD prevalence for adults and children. Subgroups analyses for pooled prevalence of CD are presented in Table 4. Gender based prevalence of biopsy-confirmed CD in not at-risk group was reported in 15 studies. Pooled prevalence of biopsy-confirmed $\mathrm{CD}$ in not at-risk group in males and females were $0.53 \%\left(95 \% \mathrm{CI} 0.3-0.7 \%, \mathrm{I}^{2}=75.5 \%, P<0.001\right)$ and $0.74 \%$ (95\% CI $\left.0.5-0.9 \%, \mathrm{I}^{2}=48.3 \%, P<0.001\right)$, respectively, and it was significantly higher in females than that in males $(P=0.04)$. The test of heterogeneity showed significant heterogeneity in the prevalence of CD in males and not in females (Table 4).

Geographical difference in the prevalence of $C D$ in low risk group. Of the 19 studies among low risk population in Asia-Pacific region, 13 studies were from Middle-East, 3 from South-Asia, 2 from Oceania, and 1 study from East-Asia. Pooled prevalence of CD in low risk group in Oceania was $0.61 \%$ (95\% CI $0.001-20 \%, \mathrm{I}^{2}=91.9 \%$, $P=0.001$ ), in the Middle-East was $0.59 \%$ (95\% CI $\left.0.4-0.7 \%, \mathrm{I}^{2}=48.8 \%, P<0.001\right)$, in the South-Asia was $0.87 \%$ (95\% CI $\left.0.4-1.5 \%, \mathrm{I}^{2}=88.8 \% \mathrm{P}<0.001\right)$ and in the East-Asia was $0.05 \%$ (95\% CI $\left.0.00-0.2 \%\right)$. Pooled prevalence among these regions, had statistically significant difference $(\mathrm{P}<0.001)$ (Table 4$)$. 
A

Study

Cook 2000 New Zealand

Hovell 2001 Australia

Shamir 2001 Israel

Shahbazkhani 2003 Iran

Israeli 2003 Israel

Tatar 2003 Turkey

Saberi-Firouzi 2003 Iran

Akbari 2003 Iran

Sood 2003 India

Ertekin 2005 Turkey

Bahari 2007 Iran

Dalgic 2008 Turkey

Farahmand 2008 Iran

Makharia 2009 India

Makharia 2009 India

Makharia 2009 India

Bhattacharya 2009 India

Sezgin 2013 Turkey

Dehghani 2013 Iran

Hatlani 2014 Saudi Arabia

Fukunaga 2016 Japan

Random effects model

Heterogeneity: $I^{2}=84 \%, \tau^{2}=0.0005, p<0.01$
Prevalence

$\begin{array}{ll}0.01 & {[0.01 ; 0.02]} \\ 0.00 & {[0.00 ; 0.00]} \\ 0.01 & {[0.00 ; 0.01]} \\ 0.01 & {[0.00 ; 0.01]} \\ 0.01 & {[0.00 ; 0.02]} \\ 0.01 & {[0.00 ; 0.01]} \\ 0.00 & {[0.00 ; 0.01]} \\ 0.01 & {[0.01 ; 0.01]} \\ 0.00 & {[0.00 ; 0.01]} \\ 0.01 & {[0.00 ; 0.01]} \\ 0.01 & {[0.01 ; 0.01]} \\ 0.00 & {[0.00 ; 0.01]} \\ 0.00 & {[0.00 ; 0.01]} \\ 0.01 & {[0.01 ; 0.01]} \\ 0.01 & {[0.01 ; 0.01]} \\ 0.01 & {[0.01 ; 0.02]} \\ 0.01 & {[0.00 ; 0.03]} \\ 0.00 & {[0.00 ; 0.01]} \\ 0.01 & {[0.00 ; 0.01]} \\ 0.01 & {[0.00 ; 0.02]} \\ 0.00 & {[0.00 ; 0.00]}\end{array}$

$\mathbf{0 . 0 1}$

$[0.00 ; 0.01]$

B

Study

Gale 1997 Australia

Shahbazkhani 2001 Iran

Imanzadeh 2003 Iran

Moayeri 2004 Iran

Aygun 2005 Turkey

Alanay 2005 Turkey

Sari 2006 Turkey

Sari 2007 Turkey

Guliter 2007 Turkey

Bhadada 2008 India

Wang 2008 China

Rostami Nejad 2008 Iran

Sharifi 2008 Iran

Pham -Short 2009 Australia

Bhat 2009 India

Al-Qabandi 2010 Kuwait

Mehrdad 2010 Iran

Srivastava 2010 India

Al-Hussaini 2010 Saudi Arabia

Saadah 2011 Saudi Arabia

Mahmoodi 2012 Iran

Watanabe 2012 Japan

Al-Sinani 2012 Oman

Dogan 2012 Turkey

Dogan 2013 Turkey

Honar 2013 Iran

Mishra 2014 India

Fukunaga 2016 Japan

Yildirmas 2016 Turkey

Random effects model

Heterogeneity: $I^{2}=76 \%, \tau^{2}=0.0038, p<0.01$

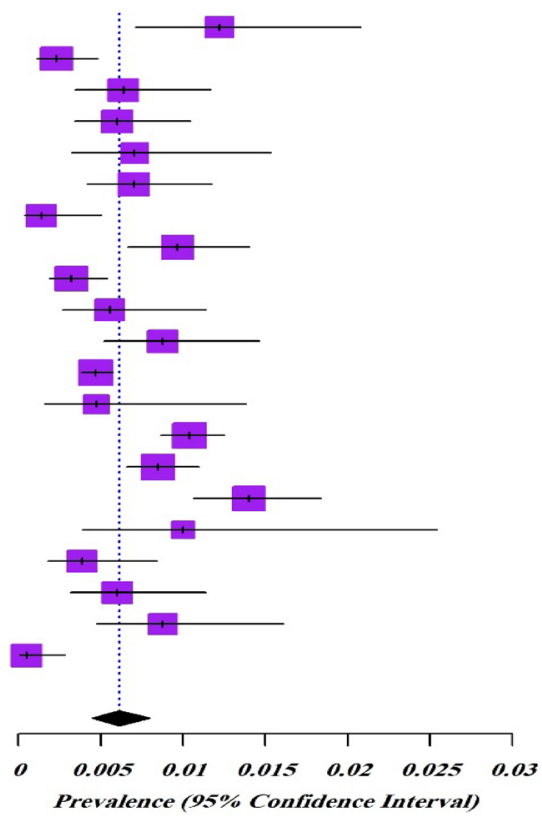

\begin{tabular}{|c|c|}
\hline Prevalence & $95 \% C I$ \\
\hline 0.04 & {$[0.01 ; 0.12]$} \\
\hline 0.02 & {$[0.01 ; 0.05]$} \\
\hline 0.07 & {$[0.05 ; 0.08]$} \\
\hline 0.03 & {$[0.01 ; 0.10]$} \\
\hline 0.02 & {$[0.01 ; 0.07]$} \\
\hline 0.01 & {$[0.00 ; 0.05]$} \\
\hline 0.05 & {$[0.02 ; 0.11]$} \\
\hline 0.06 & {$[0.02 ; 0.17]$} \\
\hline 0.06 & {$[0.03 ; 0.11]$} \\
\hline 0.11 & {$[0.07 ; 0.16]$} \\
\hline 0.12 & {$[0.07 ; 0.19]$} \\
\hline 0.02 & {$[0.01 ; 0.04]$} \\
\hline 0.08 & {$[0.04 ; 0.15]$} \\
\hline 0.04 & {$[0.04 ; 0.05]$} \\
\hline 0.06 & {$[0.03 ; 0.12]$} \\
\hline 0.06 & {$[0.02 ; 0.17]$} \\
\hline o.oo & {$[0.00 ; 0.02]$} \\
\hline 0.04 & {$[0.02 ; 0.11]$} \\
\hline 0.11 & {$[0.07 ; 0.19]$} \\
\hline 0.02 & {$[0.00 ; 0.10]$} \\
\hline 0.06 & {$[0.04 ; 0.07]$} \\
\hline 0.01 & {$[0.00 ; 0.03]$} \\
\hline 0.05 & {$[0.02 ; 0.12]$} \\
\hline 0.05 & {$[0.03 ; 0.07]$} \\
\hline 0.02 & {$[0.01 ; 0.04]$} \\
\hline 0.05 & {$[0.02 ; 0.12]$} \\
\hline 0.05 & {$[0.03 ; 0.08]$} \\
\hline 0.02 & {$[0.00 ; 0.11]$} \\
\hline 0.05 & {$[0.03 ; 0.09]$} \\
\hline 0.04 & {$[0.03 ; 0.06]$} \\
\hline
\end{tabular}

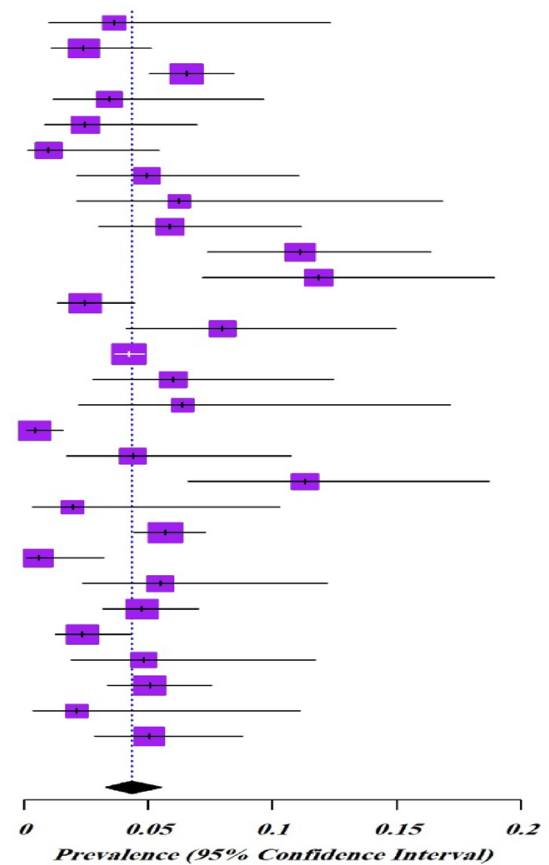

$[0.01 ; 0.07 \mid$

$0.02 ; 0.111$

0.02;0.17]

$0.03 ; 0.111$

$[0.07 ; 0.16]$

[0.01; 0.04]

$0.04 ; 0.15 \mid$

$04 ; 0.051$

$[0.03 ; 0.12]$

$[0.00 ; 0.02]$

0.02; 0.11]

$0.07 ; 0.19]$

$[0.00 ; 0.10]$

[0.00; 0.03$]$

0.02; 0.12$]$

0.03;0.07]

$[0.02 ; 0.12 \mid$

[0.03; 0.09$]$

0.04

Figure 3. Forest plot for pooled prevalence of $\mathrm{CD}$ in Asia-Pacifioc region among (A) not at-risk population, (B) at-risk population.

Pooled prevalence of biopsy-confirmed CD in high risk population. Based on the inclusion criteria, we found twenty-nine studies that reported CD prevalence in high risk population in the Asia-Pacific region $^{33-61}$ (Table 3). These studies included 10,719 subjects and of them 482 subjects were recognized with CD. Therefore, the pooled prevalence of biopsy-proven CD among at-risk population was $4.3 \%$ (95\% CI 3.3-5.5\%, $\left.\mathrm{I}^{2}=76 \%, P<0.01\right)$ (Fig. 3B). The $\mathrm{I}^{2}$ test indicated significant heterogeneity among the studies. 


\begin{tabular}{|c|c|c|c|c|c|c|c|c|c|c|c|c|}
\hline \multirow[b]{2}{*}{ First author } & \multirow[b]{2}{*}{ Country } & \multirow[b]{2}{*}{ Region } & \multirow{2}{*}{$\begin{array}{l}\text { Year of } \\
\text { study }\end{array}$} & \multirow[b]{2}{*}{ Population } & \multirow{2}{*}{$\begin{array}{l}\text { Risk } \\
\text { factor }\end{array}$} & \multirow[b]{2}{*}{ Sample size } & \multirow{2}{*}{$\begin{array}{l}\text { Age } \\
\text { (Mean) }\end{array}$} & \multirow{2}{*}{$\begin{array}{l}\text { Serology } \\
\text { tests }\end{array}$} & \multirow{2}{*}{$\begin{array}{l}\text { Risk of } \\
\text { Bias }\end{array}$} & \multicolumn{3}{|c|}{ Prevalence of biopsy-confirmed (\%) } \\
\hline & & & & & & & & & & Male & Female & Total \\
\hline Gale $^{34}$ & Australia & Pacific & 1997 & Adults & DS & 55 & 37 & AGA $^{\mathrm{a}} \mathrm{EMA}^{\mathrm{a}}$ & Moderate & - & - & $2 / 55(3.6)$ \\
\hline Pham-Short ${ }^{35}$ & Australia & Pacific & $1990-2009$ & Children & DM1 & 4,379 & 6.6 & $\begin{array}{l}\mathrm{t}-\mathrm{TG}^{\mathrm{a}} \\
\mathrm{EMA}^{\mathrm{a}}\end{array}$ & Moderate & \begin{tabular}{|l|}
$147 / 2,147$ \\
$(6.8)$
\end{tabular} & \begin{tabular}{|l|}
$38 / 2,232$ \\
$(1.7)$
\end{tabular} & $\begin{array}{l}185 / 4,379 \\
(4.2)\end{array}$ \\
\hline Imanzadeh $^{36}$ & Iran & Middle East & $1997-2003$ & Children & Diarrhea & 825 & 8.5 & AGA $^{\mathrm{a}} \mathrm{EMA}^{\mathrm{a}}$ & Moderate & $24 / 430(5.6)$ & $30 / 395(7.6)$ & $54 / 825(8.9)$ \\
\hline Al-Qabandi ${ }^{37}$ & Kuwait & Middle East & $1998-2010$ & Children & DM1 & 47 & $66 \mathrm{M}$ & $\underset{\mathrm{a}, \mathrm{b}}{\mathrm{EMA}}$ & Moderate & $1 / 16(6.2)$ & $2 / 31(6.4)$ & $3 / 47(6.4)$ \\
\hline $\begin{array}{l}\text { Shahbaz- } \\
\text { khani }^{88}\end{array}$ & Iran & Middle East & $2000-2001$ & *Both & DM1 & 250 & 18.7 & $\mathrm{EMA}^{\mathrm{a}}$ & Low & $0 / 102$ & 6/148 (4.05) & $6 / 250(2.4)$ \\
\hline Moayeri $^{39}$ & Iran & Middle East & 2003-2004 & Children & DM1 & 87 & 11.7 & $\begin{array}{l}\mathrm{t}-\mathrm{TG}^{\mathrm{a}} \\
\mathrm{EMA}^{\mathrm{a}}\end{array}$ & Moderate & $1 / 43(2.3)$ & $2 / 44(4.5)$ & $3 / 87(3.4)$ \\
\hline Bhadada $^{40}$ & India & South Asia & $2002-2008$ & *Both & DM1 & 189 & 10.8 & $\mathrm{t}-\mathrm{TG}^{\mathrm{a}}$ & Moderate & 9/93 (9.7) & $12 / 96(12.5)$ & $\begin{array}{l}21 / 189 \\
(11.1)\end{array}$ \\
\hline Aygun $^{41}$ & Turkey & Middle East & 2005 & Adults & DM1 & 122 & - & $\mathrm{EMA}^{\mathrm{a}}$ & Moderate & $1 / 54(1.8)$ & $2 / 68(2.9)$ & $3 / 122(2.4)$ \\
\hline Alanay ${ }^{42}$ & Turkey & Middle East & 2005 & Children & DS & 100 & 6.01 & $\mathrm{EMA}^{\mathrm{a}}$ & Moderate & - & - & $1 / 100(1)$ \\
\hline Sari $^{43}$ & Turkey & Middle East & 2005-2006 & Children & ATD & 101 & 12.28 & $\mathrm{t}-\mathrm{TG}^{\mathrm{a}}$ & Moderate & $0 / 11$ & $5 / 90(5.5)$ & $5 / 101(4.9)$ \\
\hline Wang $^{44}$ & China & East Asia & $2005-2008$ & Children & Diarrhea & 118 & - & $\begin{array}{l}\mathrm{EMA}^{\mathrm{a}} \\
\mathrm{t}-\mathrm{TG}^{\mathrm{a}}\end{array}$ & Low & $12 / 85(14.1)$ & $2 / 33(6.1)$ & $\begin{array}{l}14 / 118 \\
(11.8)\end{array}$ \\
\hline Sari $^{45}$ & Turkey & Middle East & 2006-2007 & ${ }^{*}$ Both & DM1 & 48 & 12.09 & $\mathrm{t}-\mathrm{TG} \mathrm{G}^{\mathrm{a}, \mathrm{b}}$ & Low & $1 / 18(5.6)$ & $2 / 30(6.7)$ & $3 / 48(6.2)$ \\
\hline Guliter $^{46}$ & Turkey & Middle East & 2006-2007 & Adults & ATD & 136 & 43.1 & $\mathrm{t}-\mathrm{TG}^{\mathrm{a}}$ & Moderate & $1 / 18(5.6)$ & $7 / 118(6)$ & $8 / 136(5.9)$ \\
\hline $\begin{array}{l}\text { Rostami } \\
\text { Nejad }^{47}\end{array}$ & Iran & Middle East & $2007-2008$ & Adults & Dyspeptic & 407 & 36.1 & $\mathrm{t}-\mathrm{TG}^{\mathrm{a}, \mathrm{b}}$ & Low & $3 / 193(1.5)$ & $7 / 214$ (3.3) & $10 / 407(2.4)$ \\
\hline Bhat $^{48}$ & India & South Asia & 2007-2009 & Children & DS & 100 & $2-18$ & $\begin{array}{l}\mathrm{EMA}^{\mathrm{a}} \\
\mathrm{t}-\mathrm{TG}^{\mathrm{a}}\end{array}$ & Moderate & - & - & $6 / 100(6)$ \\
\hline Saadah $^{49}$ & $\begin{array}{l}\text { Saudi } \\
\text { Arabia }\end{array}$ & Middle East & $2007-2011$ & Children & DS & 51 & 3.58 & $\mathrm{t}-\mathrm{TG}^{\mathrm{a}}$ & Moderate & - & - & $1 / 51(2)$ \\
\hline Sharifi ${ }^{50}$ & Iran & Middle East & 2008 & *Both & DM1 & 100 & 21.8 & $\mathrm{t}-\mathrm{TG}^{\mathrm{a}}$ & Moderate & 3/42 (7.1) & 5/58 (8.6) & $8 / 100(8)$ \\
\hline Mehrdad $^{51}$ & Iran & Middle East & $2008-2010$ & *Both & ATD & 454 & 39.4 & $\begin{array}{l}\mathrm{t}-\mathrm{TG}^{\mathrm{a}} \\
\mathrm{EMA}^{\mathrm{a}}\end{array}$ & Low & $0 / 49$ & 2/405 (0.5) & $2 / 454(0.4)$ \\
\hline Srivastava $^{52}$ & India & Middle East & 2008-2010 & Children & FDR & 91 & 9.5 & $\mathrm{t}-\mathrm{TG}^{\mathrm{a}}$ & Moderate & - & - & \begin{tabular}{|l|}
$4 / 91(4.4)$ \\
\end{tabular} \\
\hline Al-Hussaini ${ }^{53}$ & $\begin{array}{l}\text { Saudi } \\
\text { Arabia }\end{array}$ & Middle East & 2008-2010 & Children & DM1 & 106 & 8.5 & $\begin{array}{l}\mathrm{t}-\mathrm{TG}^{\mathrm{a}} \\
\mathrm{EMA}^{\mathrm{a}}\end{array}$ & Moderate & $1 / 44(2.3)$ & $11 / 62(17.7)$ & $\begin{array}{l}12 / 106 \\
(11.3)\end{array}$ \\
\hline Mahmoodi $^{54}$ & Iran & Middle East & 2009-2012 & ${ }^{*}$ Both & IBD & 1,000 & 29 & $\mathrm{t}-\mathrm{TG}^{\mathrm{a}}$ & Moderate & $21 / 497(4.2)$ & $36 / 503(7.1)$ & $\begin{array}{l}57 / 1,000 \\
(5.7)\end{array}$ \\
\hline Watanabe ${ }^{55}$ & Japan & East Asia & 2009-2012 & Adults & IBD & 172 & 43.1 & $\begin{array}{l}\mathrm{t}-\mathrm{TG}^{\mathrm{a}} \\
\mathrm{DGP}^{\mathrm{a}}\end{array}$ & Moderate & 1/102 (1) & $0 / 70$ & $1 / 172(0.6)$ \\
\hline Mishra $^{56}$ & India & South Asia & 2009-2014 & Adults & FDR & 434 & 29.8 & $\mathrm{t}-\mathrm{TG}^{\mathrm{a}}$ & Moderate & - & - & $22 / 434(5.1)$ \\
\hline Al-Sinani $^{57}$ & Oman & Middle East & 2011-2012 & Children & DM1 & 91 & \begin{tabular}{|l|}
10.8 \\
\end{tabular} & $\mathrm{t}-\mathrm{TG}^{\mathrm{a}}$ & Moderate & 2/53 (3.8) & 3/38 (7.9) & 5/91 (5.5) \\
\hline $\operatorname{Dogan}^{58}$ & Turkey & Middle East & 2012 & Adults & FDR & 484 & - & $\mathrm{t}-\mathrm{TG}^{\mathrm{a}}$ & Moderate & - & - & $23 / 484(4.8)$ \\
\hline $\operatorname{Dogan}^{59}$ & Turkey & Middle East & 2012-2013 & Adults & DM1 & 425 & 37.6 & EMA $^{a}$ & Moderate & 7/231 (3) & 3/194 (1.5) & $10 / 425(2.3)$ \\
\hline Honar $^{60}$ & Iran & Middle East & 2013 & Children & DM1 & 83 & 10.38 & $\mathrm{t}-\mathrm{TG} \mathrm{G}^{\mathrm{a}, \mathrm{b}}$ & Moderate & $1 / 34(3)$ & $3 / 49(6.1)$ & $4 / 83(4.8)$ \\
\hline Fukunaga $^{33}$ & Japan & East Asia & 2014-2016 & Adults & Abdominal & 47 & 53 & $\begin{array}{l}\mathrm{t}-\mathrm{TG}^{\mathrm{a}} \\
\mathrm{EMA}^{\mathrm{a}}\end{array}$ & Moderate & $1 / 21(4.7)$ & $0 / 26$ & $1 / 47(2.13)$ \\
\hline Yildirmaz $^{61}$ & Turkey & Middle East & 2016 & Children & DM1 & 218 & 12.9 & $\mathrm{t}-\mathrm{TG}^{\mathrm{a}}$ & Low & $6 / 101$ & $5 / 117(4.3)$ & $11 / 218(5)$ \\
\hline
\end{tabular}

Table 3. Prevalence of biopsy-confirmed of CD in Asia-Pacific region among at-risk population. EMA Antiendomysial antibodies, $A G A$ Anti-gliadin antibodies, $t-T G$ tissue transglutaminase, DGP deamidated gliadin peptides. ${ }^{*}$ Adults and children together, DM1 Diabetes Mellitus type1, DS Down syndrome, IBD Inflammatory bowel disease, $A T D$ autoimmune thyroiditis diseases, FDR first-degree relatives. ${ }^{a} \operatorname{IgA} .{ }^{b} \operatorname{IgG}$.

Prevalence of biopsy-confirmed CD amongst specific diseases. Of the 29 studies among high risk population in Asia-Pacific region, DS had 4 studies, DM1 (13 studies), diarrhea (2 studies), ATD (3 studies), dyspeptic (one study), FDR (3 studies), IBD (2 studies) and abdominal pain (one study). The pooled prevalence of CD in patients with DS was $2.9 \%\left(95 \%\right.$ CI $\left.0.39-7.6 \%, \mathrm{I}^{2}=35.2 \%, P=0.002\right)$, with DM1 was 5\% (95\% CI 3.4-6.9\%, $\left.\mathrm{I}^{2}=64.6 \%, P=0.002\right)$, with diarrhea was $8.4 \%\left(95 \%\right.$ CI $\left.0.00-58.2 \%, \mathrm{I}^{2}=71.9 \%, P=0.002\right)$, with ATD was $2.9 \%$ (95\% CI $0.00-16.7 \%, \mathrm{I}^{2}=88.9 \%, P=0.008$ ), with dyspeptic was $2.4 \%$ (95\% CI $1.1-4.1 \%$ ), with FDR was $4.8 \%$ (95\% CI $\left.4.2-5.5 \%, \mathrm{I}^{2}=1 \%, P<0.001\right)$, with IBD was $2.6 \%\left(95 \% \mathrm{CI} 0.00-87 \%, \mathrm{I}^{2}=93.7 \%, P=0.012\right)$ and with abdominal pain was $2.1 \%$ (95\% CI $0.00-8.1 \%)$. The $\mathrm{I}^{2}$ test indicated no significant heterogeneity among the studies in patients with DS and FDR (Table 4).

Age and gender based difference in the prevalence of biopsy-confirmed CD among high risk group. Of these 29 studies nine studies have focused the prevalence of CD in adults, 14 studies in children and 6 studies included both adults and children. The pooled prevalence of CD in adults at high risk was $3.1 \%$ (95\% CI $1.8-4.6 \%$, 


\begin{tabular}{|c|c|c|c|c|c|c|c|c|c|c|c|c|}
\hline & \multicolumn{4}{|c|}{ Sero-prevalence in not at-risk population } & \multicolumn{4}{|c|}{$\begin{array}{l}\text { Biopsy-confirmed prevalence in not at-risk } \\
\text { population }\end{array}$} & \multicolumn{4}{|c|}{ Biopsy-confirmed prevalence in at-risk population } \\
\hline & N. patients & N. subjects & \begin{tabular}{|l}
$\begin{array}{l}\text { Pooled } \\
\text { prevalence }\end{array}$ \\
\end{tabular} & P-value & N. patients & N. subjects & $\begin{array}{l}\text { Pooled } \\
\text { prevalence }\end{array}$ & $P$-value & N. patients & N. subjects & $\begin{array}{l}\text { Pooled } \\
\text { prevalence }\end{array}$ & $P$-value \\
\hline \multicolumn{13}{|l|}{ Population } \\
\hline Adult & 310 & 56,707 & $1.01 \%$ & \multirow{3}{*}{0.01} & 170 & 26,738 & $0.55 \%$ & \multirow{3}{*}{0.02} & 80 & 2282 & $3.13 \%$ & \multirow{3}{*}{0.08} \\
\hline Children & 664 & 28,904 & $2.01 \%$ & & 193 & 33,118 & $0.66 \%$ & & 308 & 6396 & $5.28 \%$ & \\
\hline $\begin{array}{l}\text { Adults and } \\
\text { children }\end{array}$ & 151 & 10,488 & $1.44 \%$ & & 109 & 10,488 & $1.04 \%$ & & 97 & 1454 & $4.71 \%$ & \\
\hline \multicolumn{13}{|l|}{ Sex } \\
\hline Male & 394 & 45,074 & $0.8 \%$ & \multirow{2}{*}{0.04} & 163 & 30,345 & $0.53 \%$ & \multirow{2}{*}{0.04} & 244 & 4384 & $4.02 \%$ & \multirow{2}{*}{0.43} \\
\hline Female & 543 & 37,597 & $1.65 \%$ & & 182 & 23,592 & $0.74 \%$ & & 183 & 5021 & $4.82 \%$ & \\
\hline \multicolumn{13}{|l|}{ Region } \\
\hline Oceania & - & - & - & \multirow{4}{*}{$<0.001$} & 20 & 4075 & $0.61 \%$ & \multirow{4}{*}{$<0.001$} & 187 & 4434 & $4.22 \%$ & \multirow{4}{*}{$<0.001$} \\
\hline Middle East & \begin{tabular}{|l|l|}
647 \\
\end{tabular} & 31,452 & $1.47 \%$ & & 215 & 38,538 & $0.59 \%$ & & 233 & 5226 & $4.07 \%$ & \\
\hline South Asia & 467 & 44,869 & $1.25 \%$ & & 236 & 25,723 & $0.87 \%$ & & 49 & 723 & $7.10 \%$ & \\
\hline East Asia & 11 & 19,778 & $0.06 \%$ & & 1 & 2008 & $0.05 \%$ & & 16 & 337 & $3.65 \%$ & \\
\hline \multicolumn{13}{|l|}{ Risk of CD } \\
\hline DS & - & - & - & - & - & - & - & & 10 & 306 & $2.90 \%$ & \multirow{8}{*}{0.05} \\
\hline DM1 & - & - & - & - & - & - & - & & 274 & 6145 & $5.06 \%$ & \\
\hline $\begin{array}{l}\text { Chronic } \\
\text { diarrhea }\end{array}$ & - & - & - & - & - & - & - & & 68 & 943 & $8.43 \%$ & \\
\hline ATD & - & - & - & - & - & - & - & & 15 & 691 & $2.94 \%$ & \\
\hline Dyspeptic & - & - & - & - & - & - & - & & 10 & 407 & $2.46 \%$ & \\
\hline FDR & - & - & - & - & - & - & - & & 49 & 1009 & $4.85 \%$ & \\
\hline IBD & - & - & - & - & - & - & - & & 58 & 1172 & $2.62 \%$ & \\
\hline $\begin{array}{l}\text { Abdominal } \\
\text { pain }\end{array}$ & - & - & - & - & - & - & - & & 1 & 47 & $2.13 \%$ & \\
\hline
\end{tabular}

Table 4. Subgroup analysis for pooled prevalence of CD in Asian-Pacific region among at-risk and not at-risk populations. DM1 Diabetes Mellitus type1, DS Down syndrome, IBD Inflammatory bowel disease, ATD autoimmune thyroiditis diseases, FDR first-degree relatives.

$\left.\mathrm{I}^{2}=57.9 \% P=0.001\right)$, in children was $5.2 \%\left(95 \% \mathrm{CI} 3.7-7 \%, \mathrm{I}^{2}=57.6 \%, P=0.002\right)$ and combined both adults and children was $4.7 \%$ (95\% CI $\left.1.2-10.1 \%, \mathrm{I}^{2}=91.6 \%, P=0.008\right)$, respectively. There was no significant difference in the prevalence of $\mathrm{CD}$ amongst high risk children and adults $(3.1 \%$ vs. $5.2 \%, P=0.08)$. The $\mathrm{I}^{2}$ test indicated significant heterogeneity among the studies in three subgroups (Table 4).

The pooled prevalence of $\mathrm{CD}$ amongst high risk males and females in 22 studies in Asia-Pacific region was $4.02 \%\left(95 \%\right.$ CI $\left.2.7-5.5 \%, \mathrm{I}^{2}=68.4 \%, P=0.003\right)$ and $4.8 \%$ (95\% CI 3.3-6.5\%, $\left.\mathrm{I}^{2}=82.1 \%, P=0.004\right)$, respectively and there was and there was significant difference amongst males and females $(P=0.43)$. Heterogeneity tests indicated heterogeneity in the prevalence of CD for males was less than females (Table 4).

Geographical difference in the prevalence of $C D$ in at risk group. Of all studies included in the present metaanalysis that reported the $\mathrm{CD}$ prevalence among at- risk population, 2 studies originated from Oceania, 21 from Middle-East, 3 from South-Asia, and 3 from East-Asia. The pooled prevalence of CD were $4.2 \%$ (95\% CI $\left.3.3-5.1 \%, \mathrm{I}^{2}=1 \%, P<0.001\right)$ in Oceania, $4 \%$ (95\% CI $\left.2.9-5.3 \%, \mathrm{I}^{2}=75.2 \%, P=0.002\right)$ in Middle-East, $7.1 \%$ $\left(95 \%\right.$ CI $\left.1.3-16.7 \%, \mathrm{I}^{2}=70.4 \%, P=0.002\right)$ in South-Asia and 3.6\% (95\% CI $\left.0.00-27.7 \%, \mathrm{I}^{2}=90.7 \%, P=0.017\right)$ in East-Asia (Table 4). The pooled prevalence of CD was significantly higher in high risk people in South-Asia as compared with the other region $(P=0.01)$. There were no other significant differences in the prevalence of CD in other regions. The $\mathrm{I}^{2}$ test indicated significant heterogeneity among the studies in Middle-East, South-Asia and East-Asia.

Exploration of heterogeneity. We performed meta-regression analysis to find the source of heterogeneity among the studies in low and high risk population (Table 5). The year of the study, sample size of each study, age and gender of the subjects were used for exploration the heterogeneity. While there was an inverse association between age of the participant and the prevalence of CD in low risk group of patients $(P=0.02)$, no such association was observed in high risk population. In addition, we performed meta-analysis according to risk of bias for studies including low and moderate risk. Pooled prevalence of CD according to risk of bias and also heterogeneity test are shown in Table 6.

Publication bias. The result of Egger test showed presence of publication bias for studies conducted on healthy population $(P=0.009)$ and also showed presence of publication bias for studies conducted on at-risk 


\begin{tabular}{|l|l|l|l|l|l|l|}
\hline \multirow{2}{*}{} & \multicolumn{3}{|l|}{ Not at-risk population } & \multicolumn{3}{l|}{ At-risk population } \\
\cline { 2 - 7 } & Coefficient & SE & P-value & Coefficient & SE & P-value \\
\hline Year of study & 0.0016 & 0.003 & 0.556 & 0.00 & 0.0001 & 0.656 \\
\hline Sample size & 0.00 & 0.00 & 0.497 & 0.00 & 0.00 & 0.856 \\
\hline Age & -0.0020 & 0.0007 & $0.016^{*}$ & 0.00 & 0.00 & 0.169 \\
\hline Gender & 0.004 & 0.002 & $0.042^{*}$ & 0.00 & 0.00 & 0.433 \\
\hline
\end{tabular}

Table 5. Meta-regression analysis for exploring heterogeneity among studies.

\begin{tabular}{|l|l|l|l|l|l|l|l|}
\hline & \multicolumn{4}{|l|}{ Not at-risk population } & At-risk population \\
\cline { 2 - 7 } & N. study & Pooled prevalence (95\% CI) & $\mathbf{I}^{\mathbf{2}}$ & N. study & Pooled prevalence (95\% CI) & $\mathbf{I}^{\mathbf{2}}$ \\
\hline Risk of bias \\
\hline Moderate & 15 & $0.01 \%(0.00,0.01)$ & $90.52 \%$ & 23 & $0.05 \%(0.04,0.06)$ & $76.84 \%$ \\
\hline Low & 5 & $0.01 \%(0.00,0.01)$ & $79.03 \%$ & 6 & $0.03 \%(0.01,0.06)$ & $84.26 \%$ \\
\hline
\end{tabular}

Table 6. Pooled prevalence of CD according to risk of bias.
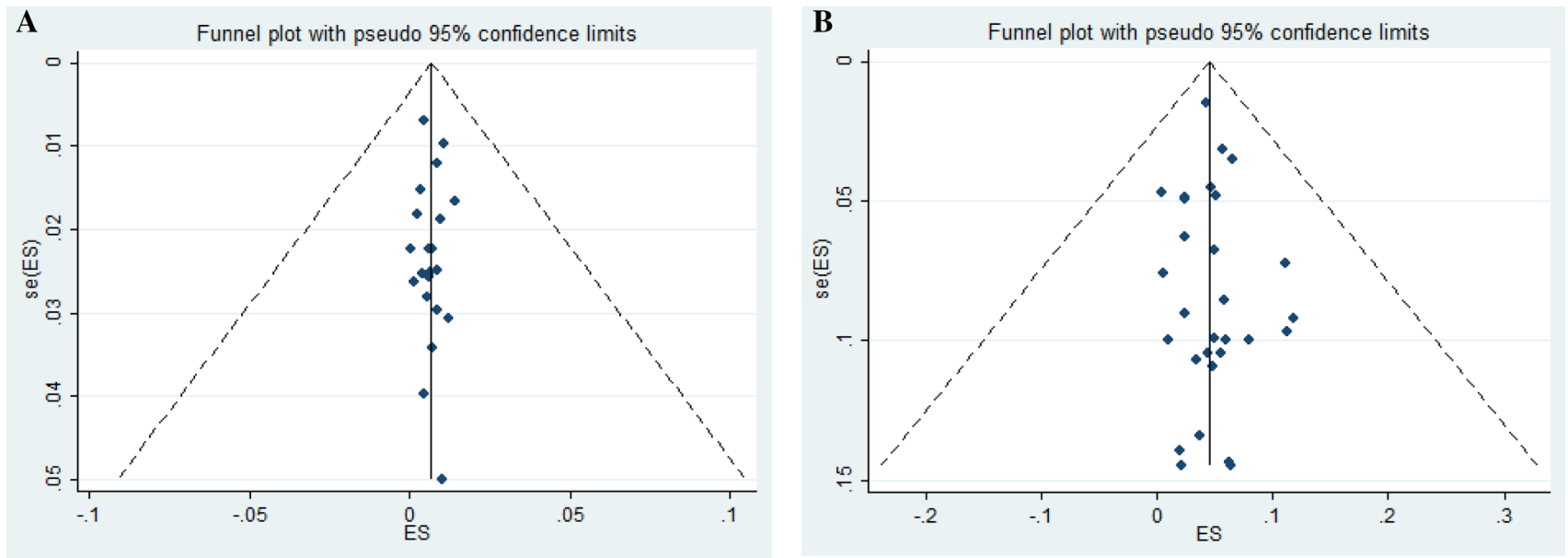

Figure 4. Funnel plot in not at-risk group (A) and at-risk group (B).

population $(P=0.003)$. Funnel plots have shown asymmetric mood and confirmed presence of publication bias (Fig. 4).

\section{Discussion}

To our knowledge, this is the first meta-analysis to examine the prevalence of CD in the Asia-Pacific region and to compare it between low and high risk groups. Considering that two previous studies in this region have shown only the prevalence of $\mathrm{CD}$ in the general population ${ }^{4,9}$, our findings represent the best approximation of the pooled prevalence of $\mathrm{CD}$ in low and high risk groups according to age (adult and children), gender (male and female) and geographical categories (Oceania, Middle-East, East-Asia, and South-Asia) in the Asia-Pacific region.

Our results revealed that the pooled sero-prevalence of CD among general population was (1.2\%), and the pooled prevalence of biopsy-confirmed CD in high risk and low risk groups was (4.3\%) and (0.61\%) respectively. So, the pooled prevalence of $\mathrm{CD}$ was significantly higher in high risk population compared to low risk subjects $(P<0.001)$. Sero-prevalence and biopsy-confirmed prevalence in Asian-Pacific countries varied from $0.06 \%$ in Turkey to $(2.8 \%)$ in Saudi Arabia and $(0.05 \%)$ in Japan and $1.4 \%$ in India, respectively. The analysis of CD prevalence within 4 geographical categories of Oceania, Middle-East, East-Asia, and South-Asia showed the highest prevalence of CD among low and high risk population was in the South-Asia $(0.8 \%)$ and (7.1\%), respectively. While, the highest sero-prevalence of CD was reported in Middle-East countries (1.4\%).

Our findings suggest that $\mathrm{CD}$ is a much greater problem in the Asia-Pacific region than has previously been appreciated. The prevalence of CD in this region, both in low and high risk groups, was similar and comparable to its prevalence in Europe and the United States ${ }^{62-64}$. Our results showed that the pooled prevalence of CD among FDR (4.8\%) in 1009 individual, were similar to those reported in previous studies in the US and Europe between $(4.5 \%)$ and $(10 \%)^{64-68}$. We found that the prevalence of CD in ATD patients was higher compared with the general 
population (2.9\% vs. $0.6 \%)$ and that the risk of CD can be increased by about $4-5$ times in ATD subjects. This is slightly higher than global pooled prevalence of biopsy proven $\mathrm{CD}(1.6 \%)$ reported by Roy et al ${ }^{69}$ in 6024 subjects with $\mathrm{ATD}^{69}$. The pooled prevalence of CD among patients with DM1 in this study was (5\%) that extracted from 13 studies on 6,145 patients with DM1. A study carried out in Sweden reveled the globally pooled prevalence of CD in DM1 patients was (6\%) based on 27 studies on $26,605 \mathrm{DM} 1$ patients ${ }^{70}$. In addition, they reported the pooled prevalence of CD among DM1 patients; (6.1\%) in Europe, (4.8\%) in North America and (4.8\%) in four countries in Middle-East and Oceania (Saudi Arabia, Iran, India and Australia) ${ }^{70}$. Our results were slightly lower than in European countries but similar to North America. Furthermore, the pooled prevalence of CD among subjects with DS (2.9\%) in this study was lower than pooled CD prevalence in DS patients that reported by Du et al. (5.8\%) based on 31 studies from Europe (21 studies), United States (6 studies) and Asia-Pacific region (4 studies) in 4383 individuals ${ }^{71}$. The pooled prevalence of CD among DS patients in the study by Du et al. was (6\%) in Europe, (5.7\%) in America and (4.5\%) in Asia-Pacific countries (India, Australia, Saudi Arabia and Israel) ${ }^{71}$.

In addition, we evaluated the prevalence of $\mathrm{CD}$ in children and adults with symptoms associated with $\mathrm{CD}$ incudes, diarrhea and abdominal pain. The pooled prevalence of CD in patients with chronic diarrhea estimated $8.4 \%$ in the study based on two studies from Iran and China on 942 children $^{36,44}$. According to the data presented in the study, CD is common among patients labeled as chronic diarrhea especially in children. Given that CD may be missed or diagnosed late in children with chronic diarrhea, immunological screening with the subsequent morphologic study of the small intestine is recommended to all patients with the chronic diarrhea syndrome to enable the early diagnostics of $\mathrm{CD}^{72}$.

Our analyses revealed a significant heterogeneity in prevalence of $\mathrm{CD}$ among low and high risk groups from different countries in Asia-Pacific region. To explore this heterogeneity we examined subgroups of studies such as year of study, sample size, age and gender. Meta-regression analysis has confirmed that CD prevalence in low risk groups decreased with age at testing and female gender. While, in high risk population did not found any association between age or gender and prevalence of CD in Asia-Pacific region. The prevalence of CD in high risk adults was significantly higher than in children, suggesting a link between the duration of gluten consumption and the development of an immune response to gluten. Therefore, heterogeneity was substantially reduced when sero-prevalence and prevalence of CD in not at-risk populations was calculated separately for men/women and adult/children. The heterogeneity reported in the prevalence of CD in this study is partly due to methodological differences between studies which include the type of diagnostic (serology/biopsy test) and study population (adults/children). It is likely that prevalence of CD also varies from country to country in Asia-Pacific, because of diverse dietary practices and prevalence of predisposing HLA-DQ2/HLA-DQ8 haplotypes in the general population $^{9,73}$.

While the present study reports a pooled prevalence of CD in Asian-Pacific region among low and high risk population for the first time, this meta-analysis has a few limitations too. Studies on the prevalence of CD in general population are available only from 13 countries in this region. Therefore, the lack of population-based prevalence data from many countries (Azerbaijan, Kazakhstan, Turkmenistan, Kyrgyzstan, Tajikistan, Cambodia, Vietnam, Mongolia, Hong Kong, Sri Lanka, Myanmar, Maldives, Nepal, and Bhutan) in the Asia-Pacific region is a major limitation. Another limitation was the most studies in this region reported the prevalence of CD based on the serology and even if the biopsies were performed in seropositive individuals, only small proportion of patients underwent biopsies. So, we had to exclude a lot of studies based on our inclusion and exclusion criteria.

In conclusion, we have undertaken the first meta-analysis study in low and high risk population in the Asia-Pacific region. Our results suggest that CD is common in Asian-Pacific region and pooled sero-prevalence and prevalence of biopsy-confirmed CD in low risk groups was $1.2 \%$ and $0.6 \%$, respectively, which is similar to Western countries. In addition, the prevalence of CD in high risk population was significantly higher than low risk group $(4.3 \%$ vs. $0.6 \% \mathrm{P}<0.001)$. High risk individuals of $\mathrm{CD}$ are key group that should be specifically targeted for prevention and control measures, and screening may prove to have an optimal cost-benefit ratio.

\section{Methods}

We developed a protocol, including eligibility criteria, search strategies, criteria for study selection and methods for extracting data according to the Preferred Reporting Items for Systematic Review and Meta-Analysis (PRISMA) guidelines ${ }^{74}$.

Search strategy. Previously published papers indexed in Medline (National Library of Medicine), PubMed, Scopus, Web of Science (Thomson Reuters; New York, USA), and Cochrane Library (Cochrane Collaboration; Oxford, United Kingdom) were searched for this systematic review and meta-analysis with the following MeSH terms and keywords: "Celiac diseases", "Coeliac disease" and "Prevalence" alone or combination. To find prevalence of $\mathrm{CD}$ among high risk population search strategy was based on the words of CD prevalence in patients with "diabetes mellitus type 1", "chronic diarrhea", "autoimmune thyroid disease", "Down syndrome", "inflammatory bowel disease", "dyspepsia", and "first-degree relatives with CD". Each one was cross-referenced with "Asia-Pacific region" and countries in this region such as Australia, New Zealand, India, Pakistan, Turkey, Iran, etc. The first recommendations for diagnosis of CD were published by the European Society for Pediatric Gastroenterology Hepatology and Nutrition (ESPGHAN) in $1990^{75}$, which we considered this year as a dividing year for well-defined diagnostic criteria for CD and other gluten-related disorders. All related articles published between January 1991 and March 2018 were, therefore, included in this review. The search for the studies was performed in English and this analysis did not include those without access to the full text. Moreover, in order to conclude the qualifying studies, all reference lists of relevant publications were also reviewed and the retrieved references were also disregarded due to duplication. To exclude unrelated studies with no eligibility requirements, the names, abstracts, as well as full texts were carefully read. 
Countries covered. Asian-Pacific is a region of the world in or near the Western Pacific Ocean. The region varies in area depending on which context, but it typically includes much of East Asia, South Asia, Southeast Asia, Central Asia, Oceania and Pacific. To cover the entire Asia in this study, West Asia was also examined in this paper. Therefore, based on our purpose, Asian-Pacific region was divided into 5 sub-regions; East Asia (China, Mongolia, North Korea, South Korea, and Japan), South-Central Asia (Tajikistan, Uzbekistan, Kazakhstan, Turkmenistan, Kyrgyzstan, Sri Lanka, Bangladesh, India, Afghanistan, Pakistan, Islamic Republic of Iran, Bhutan, Nepal, and the Maldives), Southeast Asia (Brunei, Cambodia, Indonesia, Laos, Malaysia, Myanmar, Philippines, Singapore, Thailand, and Vietnam), West Asia (Georgia, Armenia, Azerbaijan, Turkey, Cyprus, Syria, Lebanon, Israel, Palestine, Jordan, Iraq, Kuwait, Bahrain, Qatar, Saudi Arabia, United Arab Emirates, Oman, and Yemen), and Oceania (Australia and New Zealand).

Diagnostic criteria for CD. The diagnosis of CD was based on a combination of at least one positive celiacspecific serological tests such as anti-tissue transglutaminase (anti-t-TG) antibodies, anti-endomysial antibodies (EMA) and deamidated gliadin peptides (DGP) antibodies, anti-gliadin antibody (AGA) and all confirmation villous atrophy by duodenum biopsy according to Marsh classification ${ }^{76}$. In addition, studies that reporting the sero-prevalence of CD in the healthy population (having a positive t-TG, EMA and DPG antibodies without biopsy confirmation) were analyzed separately.

Inclusion criteria. For evaluating the prevalence of CD; all population based studies reporting the prevalence of CD in not at-risk population and hospital registries studies for at-risk population in Asia-Pacific region were recorded.

Exclusion criteria. The exclusion criteria were as following: (a) studies documenting the prevalence based on self-reporting (b) studies that reporting the CD prevalence by only (AGA) marker (c) Case Report, Case Series and Letter to Editor Studies were excluded (d) studies without access to the full text and those with unclear results were excluded.

Study selection and quality assessment. Two authors (A.S and NM.H) performed the literature search, reviewed all the full texts, and individually evaluated the articles based on pre-decided inclusion and exclusion criteria. Moreover, the risk of bias was calculated using the risk of bias tool for prevalence studies developed by Hoy et al. ${ }^{77}$. Based on this tool, studies were assessed for external and internal validity using a 10-point checklist and grouped into a low, moderate, or high risk of bias. The studies with a score of less than 6 were considered to have a high risk, 6 to 8 was considered a moderate risk, and 9 to 10 was considered a low risk of bias. The studies with a high risk of bias were excluded from the present meta-analysis. Disagreements between two authors were resolved by discussion. In case disagreements persisted, third author (RN.M.) reviewed the study and made the final decision. To increase the quality of the review, a blind method was used hiding the authors name and name of the journal.

Data extraction. Information was extracted separately about the sero-prevalence and biopsy confirmed prevalence of $\mathrm{CD}$ in at-risk and not at-risk populations in adults and children. Information contained the name of the first author, year of publication, place of study, demographic characteristics of study participants including; number, sex and age, the type of serological tests and duodenal biopsy. Based on our inclusion criteria, finally 61 articles including 19 articles on CD prevalence in not at-risk population, 29 on CD prevalence among at-risk population and 13 articles on sero-prevalence of $\mathrm{CD}$ in not at-risk population in English language from January 1991 to March 2018, which reported the prevalence or sero-prevalence of CD in Asia-Pacific region, were entered in this study.

Pooled prevalence and sero-prevalence of CD. Only studies in which 50 percent or more of seropositive individuals (those with positive anti-tTG and/or AEA) underwent a biopsy were included to measure the pooled prevalence of $\mathrm{CD}$. The 50 percent discontinuity value was chosen because we assumed that the real prevalence of biopsy-proven CD was wrongly reduced among the studies in which less than 50 percent of positive individuals were subjected to biopsy. For the estimation of pooled sero-prevalence only, studies in which less than $50 \%$ of seropositive individuals underwent a biopsy were included.

Statistical analysis. We obtained pooled prevalence and sero-prevalence of CD in not at-risk and at-risk population, separately. Pooled prevalence of CD was obtained based on the proportion of individuals with CD and its confidence interval in each study. Prevalence was calculated assuming binomial distribution. In addition, we calculated prevalence of CD for subgroups such as region or sex. CD prevalence between groups was compared using chi-square test. For all pooled prevalence, the random model was used. $\mathrm{I}^{2}$ statistics was employed to evaluate heterogeneity among studies. $\mathrm{I}^{2}$ value $>50 \%$ was denoted as high heterogeneity. We applied the fixed effect model when the data were homogeneous. When the cause of heterogeneity was not known, the random effect model was used. To explore the sources of heterogeneity, meta-regression analysis was done. Moreover, Begg's test was carried out for recognizing publication bias. All analyzes performed by STATA 14.0 (STATA Corp; College Station, Texas, USA) software and significant level was considered as 0.05 .

Received: 2 September 2020; Accepted: 14 January 2021

Published online: 27 January 2021 


\section{References}

1. Rostami Nejad, M., Hogg-Kollars, S., Ishaq, S. \& Rostami, K. Subclinical celiac disease and gluten sensitivity. Gastroenterol. Hepatol. Bed. Bench. 4, 102-108 (2011).

2. Green, P. H. \& Jabri, B. Coeliac disease. Lancet 362, 383-391. https://doi.org/10.1016/s0140-6736(03)14027-5 (2003).

3. Fasano, A. \& Catassi, C. Clinical practice. Celiac disease. N. Engl. J. Med. 367, 2419-2426. https://doi.org/10.1056/NEJMcp1113 994 (2012).

4. Singh, P., Arora, S., Singh, A., Strand, T. A. \& Makharia, G. K. Prevalence of celiac disease in Asia: A systematic review and metaanalysis. J. Gastroenterol. Hepatol. 31, 1095-1101. https://doi.org/10.1111/jgh.13270 (2016).

5. Ashtari, S. et al. Prevalence of gluten-related disorders in Asia-Pacific region: A systematic review. J. Gastrointest. Liver Dis. 28, 95-105. https://doi.org/10.15403/jgld.2014.1121.281.sys (2019).

6. Costa Gomes, R. et al. The celiac iceberg: From the clinical spectrum to serology and histopathology in children and adolescents with type 1 diabetes mellitus and Down syndrome. Scand. J. Gastroenterol. 51, 178-185. https://doi.org/10.3109/00365 521.2015.1079645 (2016).

7. Singh, P. et al. Global prevalence of celiac disease: Systematic review and meta-analysis. Clin. Gastroenterol. Hepatol. 16, 823-36. e2. https://doi.org/10.1016/j.cgh.2017.06.037 (2018).

8. Parra-Medina, R. et al. Prevalence of celiac disease in latin America: A systematic review and meta-regression. PLoS ONE 10, e0124040. https://doi.org/10.1371/journal.pone.0124040 (2015).

9. Cummins, A. G. \& Roberts-Thomson, I. C. Prevalence of celiac disease in the Asia-Pacific region. J. Gastroenterol. Hepatol. 24, 1347-1351. https://doi.org/10.1111/j.1440-1746.2009.05932.x (2009).

10. Tatar, G. et al. Screening of tissue transglutaminase antibody in healthy blood donors for celiac disease screening in the Turkish population. Digest. Dis. Sci. 49, 1479-1484 (2004).

11. Saberi-Firouzi, M., Omrani, G. R., Nejabat, M., Mehrabani, D. \& Khademolhosseini, F. Prevalence of celiac disease in Shiraz, southern Iran. Saudi J. Gastroenterol. 14, 135 (2008).

12. Ertekin, V., Selimoglu, M. A., Kardas, F. \& Aktas, E. Prevalence of celiac disease in Turkish children. J. Clin. Gastroenterol. 39, 689-691 (2005).

13. Dalgic, B. et al. Prevalence of celiac disease in healthy Turkish school children. Am. J. Gastroenterol. 106, 1512 (2011).

14. Aljebreen, A. M., Almadi, M. A., Alhammad, A. \& Al Faleh, F. Z. Seroprevalence of celiac disease among healthy adolescents in Saudi Arabia. World J. Gastroenterol. WJG. 19, 2374 (2013).

15. Abu-Zeid, Y. A., Jasem, W. S., Lebwohl, B., Green, P. H. \& ElGhazali, G. Seroprevalence of celiac disease among United Arab Emirates healthy adult nationals: A gender disparity. World J. Gastroenterol. WJG. 20, 15830 (2014).

16. Makharia, G. K. et al. Prevalence of celiac disease in the northern part of India: a community based study. J. Gastroenterol. Hepatol. 26, 894-900 (2011).

17. Yuan, J. et al. Prevalence of celiac disease autoimmunity among adolescents and young adults in China. Clin. Gastroenterol. Hepatol. 15, 1572-1579 (2017).

18. Sezgin, O., Sarıtaş, B., Aydın, İ, Şaşmaz, T. \& Lınked, E. Celiac disease prevalence in Turkey: A population based cross-sectional study. Acta Med. 32, 463 (2016).

19. Ramakrishna, B. et al. Prevalence of adult celiac disease in India: Regional variations and associations. Am. J. Gastroenterol. 111, 115 (2016).

20. Dehghani, S. M., Haghighat, M., Mobayen, A., Rezaianzadeh, A. \& Geramizadeh, B. Prevalence of celiac disease in healthy Iranian school children. Ann. Saudi Med. 33, 159-161 (2013)

21. Al Hatlani, M. M. Prevalence of celiac disease among symptom-free children from the Eastern Province of Saudi Arabia. Saudi J. Gastroenterol. 21, 367 (2015).

22. Yap, T. W. C. et al. Prevalence of serum celiac antibodies in a multiracial Asian population-a first study in the young Asian adult population of Malaysia. PLoS ONE 10, e0121908 (2015).

23. Cook, H. B. et al. Adult coeliac disease: Prevalence and clinical significance. J. Gastroenterol. Hepatol. 15, 1032-1036 (2000).

24. Hovell, C. J. et al. High prevalence of coeliac disease in a population-based study from Western Australia: A case for screening?. Med. J. Aust. 175, 247-250 (2001).

25. Shamir, R. et al. The use of a single serological marker underestimates the prevalence of celiac disease in Israel: A study of blood donors. Am. J. Gastroenterol. 97, 2589 (2002).

26. Shahbazkhani, B. et al. High prevalence of coeliac disease in apparently healthy Iranian blood donors. Eur. J. Gastroenterol. Hepatol. 15, 475-478 (2003).

27. Israeli, E. et al. Prevalence of celiac disease in an adult Jewish population in Israel. IMAJ-Israel Med. Assoc. J. 12, 266 (2010).

28. Akbari, M. R. et al. Screening of the adult population in Iran for coeliac disease: comparison of the tissue-transglutaminase antibody and anti-endomysial antibody tests. Eur. J. Gastroenterol. Hepatol. 18, 1181-1186 (2006).

29. Sood, A., Midha, V., Sood, N., Avasthi, G. \& Sehgal, A. Prevalence of celiac disease among school children in Punjab, North India. J. Gastroenterol. Hepatol. 21, 1622-1625 (2006).

30. Bahari, A., Karimi, M., Sanei-Moghaddam, I. \& Firouzi, F. Prevalence of celiac disease among blood donors in Sistan and Balouchestan Province Southeastern Iran. Arch. Iran. Med. 13, 301 (2010).

31. Farahmand, F. et al. Prevalence of occult celiac disease in healthy Iranian school age children. Arch. Iran. Med. 15, 342 (2012).

32. Bhattacharya, M., Dubey, A. \& Mathur, N. Prevalence of celiac disease in north Indian children. Indian Pediatr. 46, 415 (2009).

33. Fukunaga, M. et al. Celiac disease in non-clinical populations of Japan. J. Gastroenterol. 53, 208-214 (2018).

34. Gale, L., Wimalaratna, H., Brotodiharjo, A. \& Duggan, J. M. Down's syndrome is strongly associated with coeliac disease. Gut 40, 492-496. https://doi.org/10.1136/gut.40.4.492 (1997).

35. Pham-Short, A., Donaghue, K., Ambler, G., Chan, A. \& Craig, M. Coeliac disease in type 1 diabetes from 1990 to 2009 : Higher incidence in young children after longer diabetes duration. Diabet. Med. 29, e286-e289 (2012).

36. Imanzadeh, F. et al. Celiac disease in children with diarrhea is more frequent than previously suspected. J. Pediatr. Gastroenterol. Nutr. 40, 309-311 (2005).

37. Wafaa, E. B., Al-Abdulrazzaq, D., Hamadi, K. \& Al, R. F. Celiac disease in children: Is it a problem in Kuwait?. Clin. Exp. Gastroenterol. 8, 43 (2015).

38. Shahbazkhani, B. et al. Coeliac disease in Iranian type I diabetic patients. Digest. Liver Dis. 36, 191-194 (2004).

39. Moayeri, H. \& Bahremand, S. Prevalence of celiac disease in children and adolescents with type I diabetes mellitus. Med. J. Islam. Republ. Iran. 18, 39-43 (2004).

40. Bhadada, S. K. et al. Prevalence and clinical profile of celiac disease in type 1 diabetes mellitus in north India. J. Gastroenterol. Hepatol. 26, 378-381 (2011).

41. Aygun, C. et al. Celiac disease in an adult Turkish population with type 1 diabetes mellitus. Digest. Dis. Sci. 50, 1462-1466 (2005).

42. Alanay, Y., Boduroglu, K. \& Tuncbilek, E. Celiac disease screening in 100 Turkish children with Down syndrome. Turk. J. Pediatr. 47, 138-140 (2005).

43. Sari, S., Yesilkaya, E., Egritas, O., Bideci, A. \& Dalgic, B. Prevalence of celiac disease in Turkish children with autoimmune thyroiditis. Digest. Dis. Sci. 54, 830-832 (2009).

44. Wang, X. et al. Celiac disease in children with diarrhea in 4 cities in China. J. Pediatr. Gastroenterol. Nutr. 53, 368-370 (2011). 
45. Sari, S. et al. Prevalence of Celiac disease in Turkish children with type 1 diabetes mellitus and their non-diabetic first-degree relatives. Turk. J. Gastroenterol. 21, 34-38 (2010).

46. Guliter, S. et al. Prevalence of coeliac disease in patients with autoimmune thyroiditis in a Turkish population. World J. Gastroenterol. 13, 1599 (2007).

47. Rostami Nejad, M. et al. Celiac disease in dyspeptic patients. Koomesh. 12, 209-214 (2011).

48. Bhat, A. S. et al. Prevalence of celiac disease in Indian children with Down syndrome and its clinical and laboratory predictors. Indian J. Pediatr. 80, 114-117. https://doi.org/10.1007/s12098-012-0838-1 (2013).

49. Saadah, O. I., Al-Aama, J. Y., Alaifan, M. A., Bin Talib, Y. Y. \& Al-Mughales, J. A. Prevalence of celiac disease in children with Down syndrome screened by anti-tissue transglutaminase antibodies. Saudi Med. J. 33, 208-210 (2012).

50. Sharifi, N., Khoshbaten, M., Aliasgarzade, A. \& Bahrami, A. Celiac disease in patients with type-1 diabetes mellitus screened by tissue transglutaminase antibodies in northwest of Iran. Int. J. Diabet. Dev. Countr. 28, 95 (2008).

51. Mehrdad, M. et al. Frequency of celiac disease in patients with hypothyroidism. J. Thyroid Res. 20, 12 (2012).

52. Srivastava, A. et al. Prevalence, human leukocyte antigen typing and strategy for screening among Asian first-degree relatives of children with celiac disease. J. Gastroenterol. Hepatol. 25, 319-324 (2010).

53. Al-Hussaini, A., Sulaiman, N., Al-Zahrani, M., Alenizi, A. \& El Haj, I. High prevalence of celiac disease among Saudi children with type 1 diabetes: A prospective cross-sectional study. BMC Gastroenterol. 12, 180 (2012).

54. Mahmoodi, A., Jafarihaydarlo, A., Yasemi, M., Hemati, K. \& Peyman, H. Celiac disease prevalence in the patients with irritable bowel syndrome in the Ilam province; a cross sectional study from Western Iran. J. Clin. Diagn. Res. 8, 1 (2014).

55. Watanabe, C. et al. Prevalence of serum celiac antibody in patients with IBD in Japan. J. Gastroenterol. 49, 825-834 (2014).

56. Mishra, A. et al. Prevalence of celiac disease among first-degree relatives of Indian celiac disease patients. Digest. Liver Dis. 48, 255-259 (2016).

57. Al-Sinani, S. et al. Prevalence of celiac disease in omani children with type 1 diabetes mellitus: A cross sectional study. Oman Med. J. 28, 260 (2013).

58. Dogan, Y., Yldrmaz, S. \& Özercan, I. H. Prevalence of celiac disease among first-degree relatives of patients with celiac disease. J. Pediatr. Gastroenterol. Nutr. 55, 205-208 (2012).

59. Dogan, B. et al. Prevalence of celiac disease in adult type 1 patients with diabetes. Pak. J. Med. Sci. 31, 865 (2015).

60. Honar, N., Karamizadeh, Z. \& Saki, F. Prevalence of celiac disease in patients with type 1 diabetes mellitus in the south of Iran. Turk. J. Gastroenterol. 24, 122-126 (2013).

61. Yildirmaz, S., Altay, D., Esen, I. \& Dogan, Y. Prevalence of celiac disease in children with type 1 diabetes mellitus in southeast region of Turkey. Int. J. Clin. Pediatr. 5, 32-35 (2016).

62. Fasano, A. et al. Prevalence of celiac disease in at-risk and not-at-risk groups in the United States: A large multicenter study. Arch. Intern. Med. 163, 286-292. https://doi.org/10.1001/archinte.163.3.286 (2003).

63. Hill, I. et al. The prevalence of celiac disease in at-risk groups of children in the United States. J. Pediatr. 136, 86-90. https://doi. org/10.1016/s0022-3476(00)90055-6 (2000).

64. Dube, C. et al. The prevalence of celiac disease in average-risk and at-risk Western European populations: A systematic review. Gastroenterology 128, S57-67. https://doi.org/10.1053/j.gastro.2005.02.014 (2005).

65. Lewis, N. R. \& Scott, B. B. Meta-analysis: Deamidated gliadin peptide antibody and tissue transglutaminase antibody compared as screening tests for coeliac disease. Aliment Pharmacol. Ther. 31, 73-81. https://doi.org/10.1111/j.1365-2036.2009.04110.x (2010).

66. Dolinsek, J., Urlep, D., Karell, K., Partanen, J. \& Micetic-Turk, D. The prevalence of celiac disease among family members of celiac disease patients. Wien Klin Wochenschr. 116, 8-12 (2004).

67. Rubio-Tapia, A. et al. Predictors of family risk for celiac disease: A population-based study. Clin. Gastroenterol. Hepatol. 6, $983-987$. https://doi.org/10.1016/j.cgh.2008.04.008 (2008).

68. Singh, P., Arora, S., Lal, S., Strand, T. A. \& Makharia, G. K. Risk of celiac disease in the first- and second-degree relatives of patients with celiac disease: A systematic review and meta-analysis. Am. J. Gastroenterol. 110, 1539-1548. https://doi.org/10.1038/ ajg.2015.296 (2015).

69. Roy, A. et al. Prevalence of celiac disease in patients with autoimmune thyroid disease: A meta-analysis. Thyroid. 26, 880-890. https://doi.org/10.1089/thy.2016.0108 (2016).

70. Elfstrom, P., Sundstrom, J. \& Ludvigsson, J. F. Systematic review with meta-analysis: Associations between coeliac disease and type 1 diabetes. Aliment Pharmacol. Ther. 40, 1123-1132. https://doi.org/10.1111/apt.12973 (2014).

71. Du, Y., Shan, L. F., Cao, Z. Z., Feng, J. C. \& Cheng, Y. Prevalence of celiac disease in patients with Down syndrome: A meta-analysis. Oncotarget. 9, 5387-5396. https://doi.org/10.18632/oncotarget.23624 (2018).

72. Sabel'nikova, E. A. et al. Prevalence of celiac disease in patients with chronic diarrhea. Eksp Klin Gastroenterol. 31, 102-103 (2004).

73. Makharia, G. K. Celiac disease screening in southern and East Asia. Dig Dis. 33, 167-174. https://doi.org/10.1159/000369537 (2015).

74. Moher, D., Liberati, A., Tetzlaff, J. \& Altman, D. G. Preferred reporting items for systematic reviews and meta-analyses: The PRISMA statement. Ann. Intern. Med. 151, 264-269 (2009).

75. Revised criteria for diagnosis of coeliac disease. Report of working group of European Society of paediatric gastroenterology and nutrition. Arch. Dis. Child. 65, 909-911. https://doi.org/10.1136/adc.65.8.909 (1990).

76. Rostami, K. Mucosal histopathology in celiac disease: a rebuttal of Oberhuber's sub-division of Marsh III. Gastroenterol. Hepatol. Bed Bench. 8, 99-109 (2015).

77. Hoy, D. et al. Assessing risk of bias in prevalence studies: Modification of an existing tool and evidence of interrater agreement. J. Clin. Epidemiol. 65, 934-939. https://doi.org/10.1016/j.jclinepi.2011.11.014 (2012).

\section{Acknowledgements}

This study was performed in the Gastroenterology and Liver Diseases Research Center of Shahid Beheshti University of Medical Sciences.

\section{Author contributions}

All authors contributed to this study; M.R.-N. and K.R. designed the study. M.A.P., H.A.A., M.R.T., G.K.M., and M.R.Z. contributed to the concept of the review and meta-analysis, S.A., H.N. and M.O. acquisition of data, analysis and interpretation of data, drafting the article. All authors edited and revised manuscript and approved final version of manuscript.

\section{Competing interests}

The authors declare no competing interests.

\section{Additional information}

Correspondence and requests for materials should be addressed to M.R.-N. 
Reprints and permissions information is available at www.nature.com/reprints.

Publisher's note Springer Nature remains neutral with regard to jurisdictional claims in published maps and institutional affiliations.

(c) (i) Open Access This article is licensed under a Creative Commons Attribution 4.0 International License, which permits use, sharing, adaptation, distribution and reproduction in any medium or format, as long as you give appropriate credit to the original author(s) and the source, provide a link to the Creative Commons licence, and indicate if changes were made. The images or other third party material in this article are included in the article's Creative Commons licence, unless indicated otherwise in a credit line to the material. If material is not included in the article's Creative Commons licence and your intended use is not permitted by statutory regulation or exceeds the permitted use, you will need to obtain permission directly from the copyright holder. To view a copy of this licence, visit http://creativecommons.org/licenses/by/4.0/.

(C) The Author(s) 2021 